2009

\title{
AC susceptibility studies of magnetic relaxation in nanoparticles of Ni dispersed in silica
}

V. Singh

M.S. Seehra

J. Bonevich

Follow this and additional works at: https://researchrepository.wvu.edu/faculty_publications

\section{Digital Commons Citation}

Singh, V.; Seehra, M. S.; and Bonevich, J., "AC susceptibility studies of magnetic relaxation in nanoparticles of Ni dispersed in silica" (2009). Faculty Scholarship. 44.

https://researchrepository.wvu.edu/faculty_publications/44

This Article is brought to you for free and open access by The Research Repository @ WVU. It has been accepted for inclusion in Faculty Scholarship by an authorized administrator of The Research Repository@ WVU. For more information, please contact ian.harmon@mail.wvu.edu. 


\section{ac susceptibility studies of magnetic relaxation in nanoparticles of Ni dispersed in silica}

V. Singh, M. S. Seehra, and J. Bonevich

Citation: Journal of Applied Physics 105, 07B518 (2009); doi: 10.1063/1.3073949

View online: https://doi.org/10.1063/1.3073949

View Table of Contents: http://aip.scitation.org/toc/jap/105/7

Published by the American Institute of Physics

\section{Articles you may be interested in}

Unusual enhancement of effective magnetic anisotropy with decreasing particle size in maghemite nanoparticles Applied Physics Letters 110, 222409 (2017); 10.1063/1.4984903

Size-dependent shifts of the Néel temperature and optical band-gap in NiO nanoparticles Journal of Applied Physics 114, 214307 (2013); 10.1063/1.4838915

Superparamagnetism

Journal of Applied Physics 30, S120 (1959); 10.1063/1.2185850

Co-existence of ferrimagnetism and spin-glass state in the spinel $\mathrm{Co}_{2} \mathrm{SnO}_{4}$ Journal of Applied Physics 113, 203905 (2013); 10.1063/1.4807294

Size dependence of magnetic parameters and surface disorder in magnetite nanoparticles Journal of Applied Physics 105, 07 B501 (2009); 10.1063/1.3055272

Static and dynamic magnetic properties of spherical magnetite nanoparticles Journal of Applied Physics 94, 3520 (2003); 10.1063/1.1599959

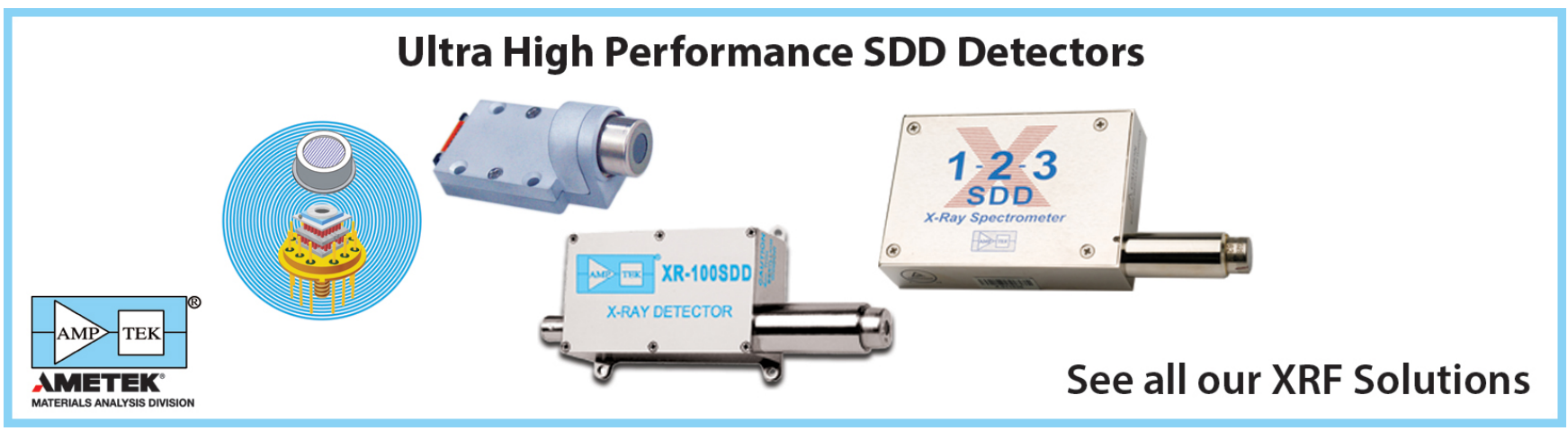




\title{
ac susceptibility studies of magnetic relaxation in nanoparticles of $\mathrm{Ni}$ dispersed in silica
}

\author{
V. Singh, ${ }^{1}$ M. S. Seehra, ${ }^{1, a)}$ and J. Bonevich ${ }^{2}$ \\ ${ }^{1}$ Department of Physics, West Virginia University, Morgantown, West Virginia 26506, USA \\ ${ }^{2}$ National Institute of Standards and Technology, Gaithersburg, Maryland 20899, USA
}

(Presented 13 November 2008; received 5 September 2008; accepted 7 December 2008; published online 25 February 2009)

\begin{abstract}
Temperature dependence of ac susceptibilities $\chi^{\prime}$ and $\chi^{\prime \prime}$ are reported using frequencies $f_{m}=0.1,1$, 99,499 , and $997 \mathrm{~Hz}$ for nanoparticles of $\mathrm{Ni}$ dispersed in silica $\left(\mathrm{Ni} / \mathrm{SiO}_{2}: 15 / 85\right)$ with the mean sizes $D=3.8,11.7,15$, and $21 \mathrm{~nm}(\sigma \simeq 0.2 \mathrm{~nm})$, as determined by transmission electron microscopy. The blocking temperatures $T_{B}$, as determined by peaks in $\chi^{\prime \prime}$ versus $T$ data, are fit to the Vogel-Fulcher law based on the following equation: $T_{B}=T_{o}+T_{a} / \ln \left(f_{o} / f_{m}\right)$. Using the attempt frequency $f_{o}=1.82$ $\times 10^{10} \mathrm{~Hz}, T_{a}(\mathrm{~K})=310(21), 954(17), 1334(14)$, and $1405(47)$ are determined for $D=3.8,11.7$, 15 , and $21 \mathrm{~nm}$, respectively, along with $T_{o}$ (representing the interparticle interaction) $=0,0$, $6.6(0.7)$, and $12.5(2.5) \mathrm{K}$ respectively. The magnitudes of $T_{a}=K_{a} V / k$ yield the anisotropy constant $K_{a}$ increasing with decreasing $D$ (or volume $V$ ) due to contributions from surface anisotropy. The validity of the theoretical result $\chi^{\prime \prime}=C \partial\left(\chi^{\prime} T\right) / \partial T$ with $C \simeq \pi /\left[2 \ln \left(f_{o} / 2 \pi f_{m}\right)\right]$ is checked and the calculated values of $f_{o}$ are consistent with experimental value of $f_{o}=1.82 \times 10^{10} \mathrm{~Hz}$. (C) 2009 American Institute of Physics. [DOI: 10.1063/1.3073949]
\end{abstract}

\section{INTRODUCTION}

Among several factors that affect the measured properties of magnetic nanoparticles (NPs) include size and size distribution, magnetic field $H$, temperature $T$, interparticle interactions (IPIs), and the time scale or frequency $f_{m}$ of measurements. ${ }^{1,2}$ The intrinsic anisotropy $K_{a}$, which is often size dependent due to different contributions from bulk anisotropy $K_{b}$ and surface anisotropy $K_{s}$, also has major effect on the observed magnetic properties. ${ }^{3-6}$ In this work, we report our investigations of the magnetic relaxation of Ni NPs (chemically dispersed in silica) with average particle diameters $D=3.8,11.7,15$, and $21 \mathrm{~nm}$ by measuring the temperature dependence of the ac susceptibilities $\chi^{\prime}$ and $\chi^{\prime \prime}$ at $f_{m}$ $=0.1,1,99,499$, and $997 \mathrm{~Hz}$. The particles were synthesized via the citric acid sol-gel route with the $\mathrm{Ni} / \mathrm{SiO}_{2}$ composition of $15 / 85$ (Ref. 7) in order to increase the interparticle separation and hence reduce the IPI.

For noninteracting NPs subjected to a slowly oscillating magnetic field $h=h_{o} \cos \omega_{m} t, \chi^{\prime}$ and $\chi^{\prime \prime}$ are given by ${ }^{2,8}$

$$
\begin{aligned}
& \chi^{\prime}=\chi_{o} /\left[1+\left(\omega_{m} \tau\right)^{2}\right], \\
& \chi^{\prime \prime}=\chi_{o} \omega_{m} \tau /\left[1+\left(\omega_{m} \tau\right)^{2}\right],
\end{aligned}
$$

where the relaxation frequency $f=1 / \tau$ is given by ${ }^{1,2}$

$$
f=f_{o} \exp \left(-T_{a} / T\right) .
$$

Here $T_{a}=K_{a} V / k$ for a particle of volume $V$ with $k$ being the Boltzmann constant, $\chi_{o}$ is the static susceptibility for $\omega \rightarrow 0$, $\omega_{m}=2 \pi f_{m}$ and $f_{o}$ is the attempt frequency. For random orientation of the easy axis of the particles each with magnetic

\footnotetext{
${ }^{a)}$ Author to whom correspondence should be addressed. Electronic mail: mseehra@wvu.edu.
}

moment $\mu=M_{s} V, \chi^{\prime}$ and $\chi^{\prime \prime}$ of Eqs. (1) and (2) can be written as ${ }^{8}$

$$
\begin{aligned}
& \chi^{\prime}=\left(M_{s}^{2} / 3 K_{a}\right)\left[1+\left(T_{a} / T\right)\left\{1 /\left[1+\left(\omega_{m} \tau\right)^{2}\right]\right\}\right], \\
& \chi^{\prime \prime}=\left(M_{s}^{2} / 3 K_{a}\right)\left[\left(T_{a} / T\right)\left\{\omega_{m} \tau /\left[1+\left(\omega_{m} \tau\right)^{2}\right]\right\}\right] .
\end{aligned}
$$

The blocking temperature $T_{B}$ of the particles is determined from Eq. (3) for $f=f_{m}$ yielding

$$
T_{B}=T_{a} / \ln \left(f_{o} / f_{m}\right) .
$$

In the presence of weak IPI, Eq. (6) is replaced by Eq. (7) below, derived from the Vogel-Fulcher law, ${ }^{9-11}$

$$
T_{B}=T_{o}+T_{a} / \ln \left(f_{o} / f_{m}\right) .
$$

Here $T_{o}$ measures the strength of IPI. According to Eq. (6), $T_{B}$ should increase with increase in $f_{m}$. Also from the above equations, it can be shown that $\chi^{\prime \prime}$ peaks at $\omega_{m} \tau=1$ and $\chi^{\prime}$ and $\chi^{\prime \prime}$ are related $\mathrm{by}^{2}$

$$
\chi^{\prime \prime}=C \partial\left(\chi^{\prime} T\right) / \partial T,
$$

where $C \simeq \pi /\left[2 \ln \left(f_{o} / 2 \pi f_{m}\right)\right]$. In this work, the above equations are used to interpret the frequency dependence of $\chi^{\prime}$ and $\chi^{\prime \prime}$ and determine $T_{B}, f_{o}$, and $T_{a}$ and their variations with the change in the size of the Ni NPs. We also check the validity of Eq. (8)

\section{EXPERIMENTAL RESULTS AND DISCUSSION}

The NPs of $\mathrm{Ni} / \mathrm{SiO}_{2}(15 / 85)$ were synthesized following the procedure outlines in an earlier paper. ${ }^{7}$ Annealing the samples at $400,500,600$, and $700{ }^{\circ} \mathrm{C}$ for $2 \mathrm{~h}$ in ultrahigh purity $\mathrm{N}_{2}$ gas produced particles of average size $D$ $=3.8(0.2), 11.7(0.2), 15(0.2)$ and $21(0.12) \mathrm{nm}$ as determined by transmission electron microscopy (TEM). In Fig. 1, we show the representative TEM for the $21 \mathrm{~nm}$ NPs with log- 


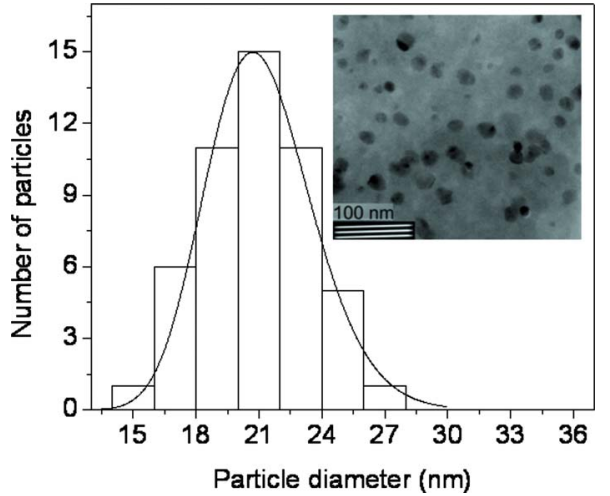

FIG. 1. (Color online) Inset shows the TEM micrograph for the $D$ $=21 \mathrm{~nm}$ sample. The solid line is a fit to log-normal distribution of the histogram of particle sizes.

normal fit to the histogram of the particle sizes. The $\mathrm{x}$-ray diffraction (XRD) patterns for all four sizes using $\mathrm{Cu} K \alpha$ source $(\lambda=0.154185 \mathrm{~nm})$ showed lines only due to fcc Ni and a broad line near $2 \theta \simeq 22^{\circ}$ due to amorphous silica. The sizes determined from the Scherrer broadening of the XRD lines were consistent with the above sizes determined from TEM. Measurements of $\chi^{\prime}$ and $\chi^{\prime \prime}$ were done using a commercial superconducting quantum interference device magnetometer with the measuring $h_{o}=7 \mathrm{Oe}$ and at frequencies $f_{m}=0.1,1,99,499$, and $997 \mathrm{~Hz}$. These measured value of $\chi^{\prime}$ and $\chi^{\prime \prime}$ were normalized to $15 \%$ concentration of $\mathrm{Ni}$ in the $\mathrm{Ni} / \mathrm{SiO}_{2}(15 / 85)$ samples

For one representative samples with size $D=21 \mathrm{~nm}$, plots of experimental $\chi^{\prime}$ and $\chi^{\prime \prime}$ versus $T$ are shown in Fig. 2. Similar data were obtained for the other three samples. It is evident that $T_{B}$ determined by the peak in $\chi^{\prime \prime}$ increases with increase in $f_{m}$, as predicted by Eqs. (6) and (7). Peaks in $\chi^{\prime}$ are broad and occur at temperatures higher than those for $\chi^{\prime \prime}$ in agreement with the prediction $\chi^{\prime \prime}=-(\pi / 2) \partial\left(\ln \chi^{\prime}\right) /$ $\partial \ln \omega .^{2}$

Using the measured values of $T_{B}$ for each $f_{m}$, we plot in Fig. $3 \ell n f_{m}$ versus $T_{B}^{-1}$ following Eq. (6) rewritten as $\ell n f_{m}$ $=\ell n f_{o}-\left(T_{a} / T_{B}\right)$. The data fit the predicted linear behavior with the intercept yielding $\ell n f_{o}$ and the slope yielding $T_{a}$. Using least-squares fitting of the data shown in Fig. 3, the magnitudes of $f_{o}$ are determined to be $2.83\left({ }_{-2.2}^{+11.6}\right) \times 10^{9} \mathrm{~Hz}$

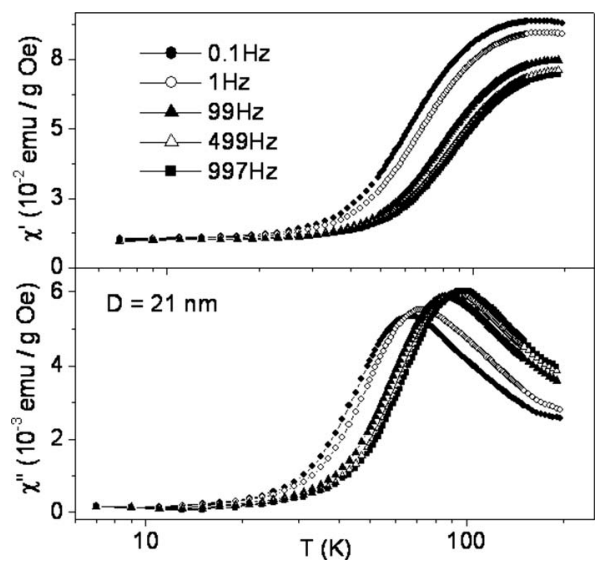

FIG. 2. Experimental $\chi^{\prime}$ and $\chi^{\prime \prime}$ vs $T$ for $D=21 \mathrm{~nm}$ at five frequencies shown.

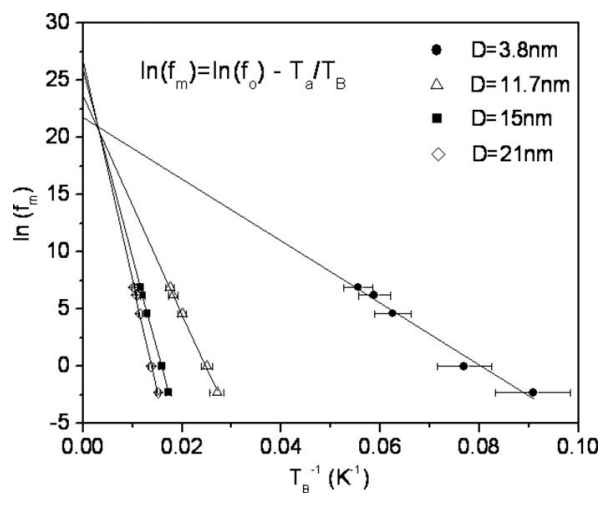

FIG. 3. Plots of $\ln f_{m}$ against $T_{B}^{-1}$ to determine $f_{o}$ and $T_{a}$ from the equation $f_{m}=f_{o} \exp \left(-T_{a} / T_{B}\right)$. The solid lines are least-squares fits to the data.

for the $D=3.8 \mathrm{~nm}$ sample and $f_{o}=1.82\left({ }_{-0.62}^{+0.94}\right) \times 10^{10} \mathrm{~Hz}, f_{o}$ $=1.72\left({ }_{-0.23}^{+0.25}\right) \times 10^{11} \mathrm{~Hz}$, and $\left.f_{o}=3.96_{-2.13}^{+4.62}\right) \times 10^{11} \mathrm{~Hz}$ for the $D=11.7 \mathrm{~nm}, D=15 \mathrm{~nm}$, and $D=21 \mathrm{~nm}$ samples, respectively. For the two larger particles, the magnitude of $f_{o}$ is higher by an order of magnitude. Since the presence of IPI often leads to increased magnitudes of $f_{o}$ (Ref. 11) when data are fit to Eq. (6), we next evaluated the parameter $\Phi$ $=\Delta T_{B} /\left[T_{B} \Delta \log _{10} f_{m}\right]$, which represents fractional change in $T_{B}$ per decade change in $f_{m}{ }^{12}$ Experiments have shown that $\Phi$ is very small (0.005-0.05) for spin glasses and $\Phi \geq 0.13$ for isolated noninteracting NPs. ${ }^{12}$ For intermediate values of $\Phi(0.05<\Phi<0.13)$, IPI is present with its effect decreasing with increase in $\Phi$. Determining $\Delta T_{B}$ for maximum and minimum $f_{m}$ in our experiments, $\Phi=0.16,0.13,0.12$, and 0.12 are found respectively for the $D=3.8,11.7,15$, and 21 $\mathrm{nm}$ samples. These magnitudes of $\Phi$ suggest the presence of a weak IPI in the larger $D=15 \mathrm{~nm}$ and $D=21 \mathrm{~nm}$ particles (possibly due to larger moment per particle) and absence of IPI in the two smaller particles. Thus it is very likely that the larger magnitudes of $f_{o}$ in the two larger NPs is due to the presence of IPI as the magnitudes of $T_{o}>0$ determined below also suggest. Taking into consideration the relatively large uncertainty in $f_{o}$ for the smallest $D=3.8 \mathrm{~nm} \mathrm{NPs}$, we have chosen $f_{o}=1.82 \times 10^{10} \mathrm{~Hz}$ for the $D=11.7 \mathrm{~nm}$ NPs as the likely magnitude for $f_{o}$ for this system. Using this magnitude of $f_{o}$, the data are plotted as $T_{B}$ against $1 / \ell n\left(f_{o} / f_{m}\right)$ in Fig. 4 to check the validity of Eq. (7). The least-squares fits are then used to determine $T_{o}$ and $T_{a}$. It is evident that $T_{o}$ is

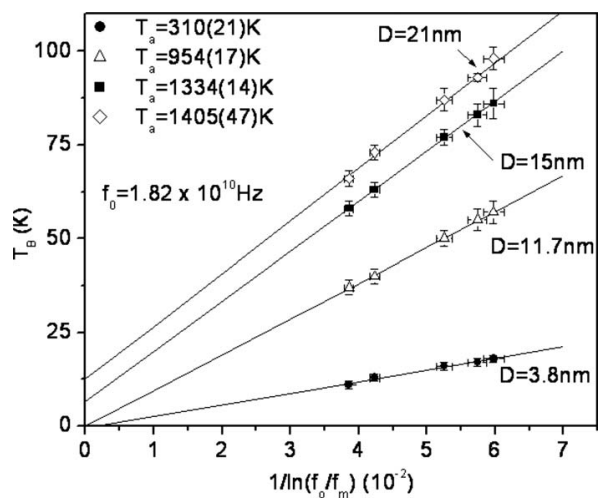

FIG. 4. Plots of $T_{B}$ vs $1 / \ln \left(f_{o} / f_{m}\right)$ following Eq. (7) for the $f_{o}$ value noted on the figure. The lines through the points are least-squares fits with magnitudes of $T_{a}$ shown. 


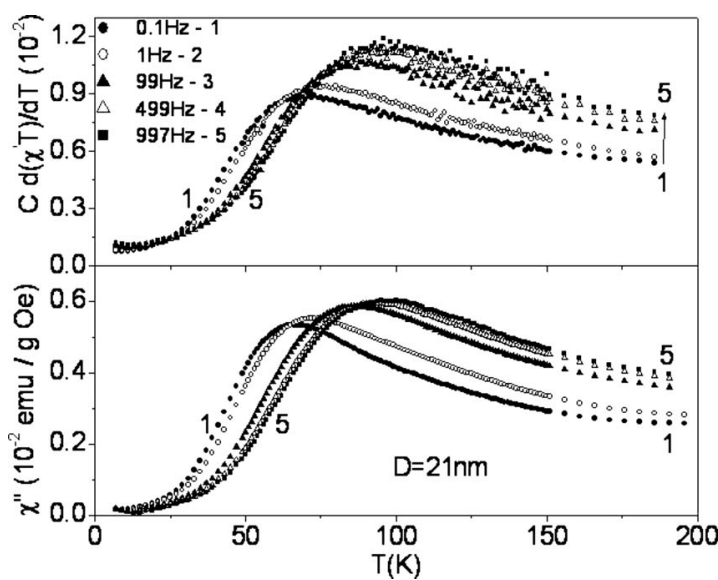

FIG. 5. Plots of experimental $\chi^{\prime \prime}$ vs $T$ and computed $C \partial\left(\chi^{\prime} T\right) / \partial T$ of Eq. (7) using the data of the $D=21 \mathrm{~nm}$ sample.

zero within experimental uncertainties for the two smaller NPs and $T_{o}=6.6(0.7) \mathrm{K}$ and $12.5(2.5) \mathrm{K}$, respectively, for the $D=15 \mathrm{~nm}$ and $D=21 \mathrm{~nm}$ samples. Also $T_{a}$ representing the energy barrier increases with increase in the size $D$ of the NPs (see Fig. 4). In an earlier publication, we reported $f_{o}$ $=2.6 \times 10^{9} \mathrm{~Hz}$ for the $D=3.8 \mathrm{~nm}$ sample using an eye-ball fit of the data ${ }^{7}$ similar to the value of $f_{o}=1.8 \times 10^{9} \mathrm{~Hz}$ reported by Goya et al. ${ }^{13}$ in a $5 \% \mathrm{Ni} / \mathrm{SiO}_{2}$ sample. This value is consistent with $f_{o}=1.82 \times 10^{10} \mathrm{~Hz}$ used here since for $f_{o}$ $=2.8\left({ }_{-2.2}^{+11.6}\right) \times 10^{9} \mathrm{~Hz}$ determined above using least-squares fitting has large enough uncertainty to accommodate $f_{o}$ $=1.82 \times 10^{10} \mathrm{~Hz}$. The important result of this analysis is that using a size independent $f_{o}$, the presence of measurable IPI is evident in the two larger sizes and $T_{a}$ increases with increase in the size of $D$ of the NPs as qualitatively expected. Later we show that the magnitude of $f_{o}=1.8 \times 10^{10} \mathrm{~Hz}$ determined above is consistent with calculations also using a theoretical expression.

Next, the magnitudes of $T_{a}=K_{a} V / k$ are used to determine $K_{a}$ representing the energy barrier assuming spherical NPs. For the cubic anisotropy of $\mathrm{Ni}, K_{a}=K_{1} / 12$, where $K_{1}$ is the first-order anisotropy constant with $K_{1}=|7.5|$ $\times 10^{5} \mathrm{ergs} / \mathrm{cm}^{3}$ for bulk Ni. ${ }^{14}$ The computed values of $\left|K_{1}\right|$ (in units of $10^{5} \mathrm{ergs} / \mathrm{cm}^{3}$ ) are 178.7, 18.8, 12.5, and 4.8 for the $D=3.8,11.7,15$, and $21 \mathrm{~nm}$ samples, respectively. The observed increase in $K_{1}$ with decreasing $D$ has been reported and discussed in other systems also, the source being increasing surface anisotropy with decreasing $D .{ }^{3-6}$ For the largest $D=21 \mathrm{~nm}$ sample, the magnitude of $K_{1}$ is close to the value for bulk Ni.

To test the validity of the correlation between $\chi^{\prime \prime}$ and $C \partial\left(\chi^{\prime} T\right) / \partial T$ predicted by Eq. (8), the plots of the experimental $\chi^{\prime \prime}$ and computed $C \partial\left(\chi^{\prime} T\right) / \partial T$ versus $T$ are shown in Fig. 5 using the $\chi^{\prime}$ versus $T$ data for our representative sample with $D=21 \mathrm{~nm}$. All primary features of experimental $\chi^{\prime \prime}$ such as the frequency and $T$ dependence, are also evident in the plots of $C \partial\left(\chi^{\prime} T\right) / \partial T$, except that the peak magnitudes are off by a factor of about 2. This discrepancy is likely related to the approximations made in deriving the magnitude of $C$ in Eq. (8). ${ }^{2}$ Comparison of $\chi^{\prime \prime}$ and computed $C \partial\left(\chi^{\prime} T\right) / \partial T$ for the other three samples yielded similar re- sults. It may be relevant to note that in bulk antiferromagnets near the Néel temperature, $\partial\left(\chi^{\prime} T\right) / \partial T$ is proportional to the specific heat. ${ }^{15}$

Next we calculate the theoretically expected magnitude of the attempt frequency $f_{o}$. Based on the earlier work by Brown, ${ }^{16}$ a simplified expression for $f_{o}$ in zero field given by Aharoni ${ }^{17}$ for the case of $T_{a} \gg T$, reduces to the following for the case of $\mathrm{Ni}$ :

$$
f_{o}=\left[T_{a} / \pi T\right]^{1 / 2} \gamma_{o} K_{1} / 6 M_{s} .
$$

Here $\gamma_{o}$ is the gyromagnetic ratio and $M_{s}$ is the saturation magnetization. For $\mathrm{Ni}$ with $g=2.2, \gamma_{o}=2 \pi g \mu_{B} / h=1.935$ $\times 10^{7}$. For $T$ in Eq. (9), we use the average $T_{B}$ measured at the frequencies $f_{m}$ used in our experiments since $f_{o}$ was determined at these temperatures and the condition $T_{a} \gg T$ is still valid using the $T_{a}$ values noted in Fig. 4 . We used the values of $M_{s}=245,216,229$, and $274 \mathrm{emu} / \mathrm{cm}^{3}$ measured at $5 \mathrm{~K}$ for the $D=3.8,11.7,15$, and $21 \mathrm{~nm}$ samples, respectively. These magnitudes of $M_{s}$ are only about $50 \%$ of the corresponding magnitude for bulk Ni, likely due to disorder of spins on the surface of a NP. For the $D=3.8 \mathrm{~nm}$ sample with $T_{a}=310 \mathrm{~K}$, Eq. (9) yields $f_{o}=6.0 \times 10^{11} \mathrm{~Hz}$. Similar calculations for the other samples yield the following $f_{o}$ values: $7.1 \times 10^{10} \mathrm{~Hz}$ for $11.7 \mathrm{~nm}, 4.2 \times 10^{10} \mathrm{~Hz}$ for $15 \mathrm{~nm}$, and $1.3 \times 10^{10} \mathrm{~Hz}$ for $21 \mathrm{~nm}$. Except for the smallest NPs, these calculated magnitudes of $f_{o}$ using Eq. (9) compare quite favorably with $f_{o}=1.8 \times 10^{10} \mathrm{~Hz}$ derived from the analysis of the data.

In summary, the variations of the magnetic relaxation parameters $T_{B}$ and $T_{a}$ with size of Ni NPs dispersed in silica matrix are shown to follow the predictions of Eqs. (1)-(8) with a size independent $f_{o}$ and the presence of a weak IPI in the larger NPs. The order of magnitude of the calculated $f_{o}$ is in agreement with the measured $f_{o}$.

The research at West Virginia University was supported in part by U.S. Department of Energy (Contract No. DE-FC26-05NT42456).

${ }^{1}$ L. Néel, Ann. Geophys. 5, 99 (1949).

${ }^{2}$ L. Lundgren, P. Svedlindh, and O. Beckman, J. Magn. Magn. Mater. 25, 33 (1981).

${ }^{3}$ F. Bødker, S. Morup, and S. Linderoth, Phys. Rev. Lett. 72, 282 (1994).

${ }^{4}$ K. Gilmore, Y. U. Idzerda, M. T. Klem, M. Allen, T. Douglas and M. Young, J. Appl. Phys. 97, 10B301 (2005).

${ }^{5}$ R. Yanes, O. C. Fesenko, H. Kachkachi, D. A. Garanin, R. Evans, and R. W. Chantrell, Phys. Rev. B 76, 064416 (2007).

${ }^{6}$ H. Shim, P. Dutta, M. S. Seehra, and J. Bonevich, Solid State Commun. 145, 192 (2008).

${ }^{7}$ V. Singh, M. S. Seehra and J. Bonevich, J. Appl. Phys. 103, $07 D 524$ (2008).

${ }^{8}$ J.-O. Andersson, C. Djurberg, T. Jonsson, P. Svedlindh, and P. Nordblad, Phys. Rev. B 56, 13983 (1997).

${ }^{9}$ S. Shtrikman and E. P. Wohlfarth, Phys. Lett. 85A, 467 (1981).

${ }^{10}$ J. L. Tholence, Solid State Commun. 88, 917 (1993).

${ }^{11}$ H. Shim, A. Mannivannan, M. S. Seehra, K. M. Reddy and A. Punnoose, J. Appl. Phys. 99, $08 Q 503$ (2006) and references therein.

${ }^{12}$ J. L. Dormann, L. Bessais, and D. Fiorani, J. Phys. C 21, 2015 (1988).

${ }^{13}$ G. F. Goya, F. C. Fonseca, R. F. Jardim, R. Muccillo, N. L. V. Carreno, E. Longo, and E. R. Leite, J. Appl. Phys. 93, 6531 (2003).

${ }^{14}$ J. I. Gittleman, B. Abeles, and S. Bozowski, Phys. Rev. B 9, 3891 (1974).

${ }^{15}$ E. E. Bragg and M. S. Seehra, Phys. Rev. B 7, 4197 (1973); M. E. Fisher, Philos. Mag. 7, 1731 (1962)

${ }^{16}$ W. F. Brown, Jr., J. Appl. Phys. 34, 1319 (1963).

${ }^{17}$ A. Aharoni, Phys. Rev. B 7, 1103 (1973). 